\title{
The applications of anisotropic electrodes in vacuum circuit breakers to increase the switching life
}

\author{
Yuri Samofalov ${ }^{1, *}$, Ilgiz Valeev $^{1}$, and Victor Maksimov ${ }^{1}$ \\ ${ }^{1}$ KSPEU, Chair: Electrical power systems and grids, 420066 Russia, Kazan, Str. Krasnoselskaya, 51
}

\begin{abstract}
The article analyzes the disadvantages of the vacuum circuit breaker, the transients that occur during switching, as well as the processes that lead to a decrease in the contact system resource. The simulation of the vacuum circuit breaker electrodes was performed using the COMSOL Multiphysics software package. The possibility of increasing the energy characteristics of vacuum circuit breakers by using single-crystal electrodes of copper, nickel, and iron as overlays is shown. A contact device for a vacuum circuit breaker with arc-resistant pads made of anisotropic materials has been developed.
\end{abstract}

\section{Introduction}

The widespread use of vacuum circuit breakers (VCB) is due to the numerous advantages of this type of circuit breakers, namely: operating speed, explosion and fire safety, no environmental pollution, wide temperature range, simplicity of operation, noiselessness, minimal application costs, relatively small overall dimensions, high resistance to shock and vibration loads, high wear resistance when switching nominal and load currents, as well as easy replacement of the vacuum arc extinguishing chamber and its arbitrary placement.

The analysis of the conducted research in the Republic of Tatarstan shows that the VCB percentage ratio is $40 \%$ of the installed high-voltage switches total number. However, despite the indisputable advantages considerable number, there have a series of their usage disadvantages.

The contact system of VCB is located directly in a high vacuum, the electrical strength of which is significantly higher than the strength of air at atmospheric pressure. When the contacts are opened, the starting jerk force on the mobile contact can reach 2000 $\mathrm{N}$, which makes it possible to effectively break the points of micro-welds on the contact surface that arise due to the thermal effect of short-circuit currents [1]. The arc itself is extinguished during the first transition of the current through zero, by means of the diffusion of charged particles from the arc region into the environment. Next, the arc begins to light up as a result of the so-called ionization process of metal contact vapors. When the contacts open, the cross-section of the contact pads begins to significantly decrease, and the resistance and temperature, in turn, on the contrary, begin to increase and plasma appears which as a result leads to melting and evaporation of the metal (Fig. 1). At the same time, erosion occurs on the surface of the contacts, the appearance of craters on them or metal sticking (cathode spots). The metal transfer is observed towards the electrode further away from the melting point, usually towards the cathode. The transferred metal hardens at the cathode in the form of sharp protrusions, which worsen the contact conditions and reduce the gap between the contacts in the open state.

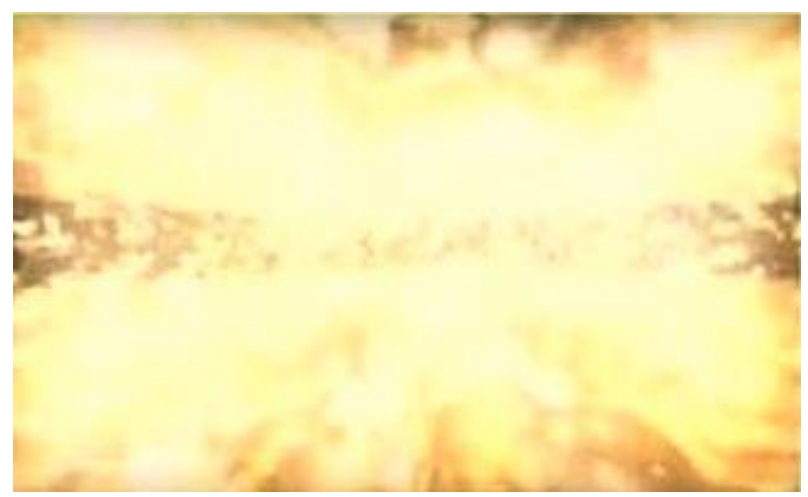

Fig. 1. Plasma in the inter-contact gap when the contacts are opened.

It should be noted that vacuum switching devices can cause significant overvoltage when switching off inductive loads (unloaded transformers, electric motors in start-up mode). Due to the special properties of the vacuum, these overvoltages may differ in nature from the overvoltages created under the same conditions by switching devices that use a different arc-extinguishing medium (air, gas or oil). When switching the electrical grid of VCB, the following processes occur: repeated arc ignition during disconnection and a virtual current slice.

The article shows the possibility of increasing the energy characteristics of vacuum circuit breakers by using single-crystal electrodes of copper, nickel, and iron as overlays. 


\section{Results and discussion}

\subsection{Re-ignitions}

Re-ignition occurs in the contact opening operation, when the electric arc is re-ignited after an interruption.

In [2], it is noted that such re-ignition usually occurs fairly soon, in the interval $(<0.1 \mathrm{~ms})$ after the arc breaks and does not leave damage, since the arc channel remains in the normal position between the switch contacts until the next zero value of the flowing current appears.

Therefore, re-ignition is useful for circuit breakers, as they provide it with an additional half-life of additional contact separation before being interrupted.

Re-ignition, as defined here, is when the arc is restored during a voltage surge to a transient restoring voltage, after exchanging heat with the arc-extinguishing environment.

According to the definition [3] dielectric breakdown arc resistance of the channel within $5 \mathrm{~ms}$ after interruption of the current is called "re-ignition", and a breakdown of dielectric breakdown arc channel in $5 \mathrm{~ms}$ aftershocks. However, in the event of a re-impact, the arc channel has already been extinguished at zero current, no re-ignition has occurred, and a new arc channel can subsequently be initiated when a re-impact occurs between two new points. In these cases, the new arc channel may never go out and may seriously damage, impair the operation of the interrupting chamber.

\subsection{Current cutoff}

A very important parameter of the VCB is the current cutoff, which affects the state of the surface of the contacting electrodes.

During the interruption of the current by the circuit breaker in the AC grid, the arc always stops before the natural zero of the current. This phenomenon is called "current cutoff". It can lead to the occurrence of high surge circuits. The source of these overvoltage's is the magnetic energy stored in the inductive load, which can be a reactor, a transformer at idle, or a "locked" motor. This energy is transferred to the capacity on the load side-usually the capacity of the cable - which is often small. The amplitude of this overvoltage caused by the current interruption can be calculated using Equation 1

$$
U_{\text {max }}=\sqrt{\left\{\left(U_{n}+U_{0}\right)^{2}+\left(i_{0} * \sqrt{\frac{L_{t}}{c_{t}}}\right)^{2}\right\}}
$$

where $L_{t}$ is the equivalent reactance of the system; $C_{t}$ is the capacitance on the load side; $i_{0}$ is equal to the interrupt current through the load; $U_{n}$ is the source voltage; $\mathrm{U}_{0}$ is the instantaneous arc voltage at the time of the interrupt.

As shown in the equation above, the value of the intermittent current determines the amplitude of the transient voltage. The value of this current in the $\mathrm{VCB}$ mainly depends on the contact material [4]. While the average cut-off current for copper contacts is $15 \mathrm{~A}$, for copper/chromium alloy contacts $(50-80 \% \mathrm{Cu}, 50-20 \%$ $\mathrm{Cr}$ ), which are widely used in many of the manufacturer's products, it is $4 \mathrm{~A}$, whereas for silver alloy $\mathrm{Ag} / \mathrm{W} / \mathrm{C}$, used in high-speed switching devices and switchgear with a long service life (such as contactors), it is $0.5 \mathrm{~A}$. With respect to the composition of the contact material of the interrupter, the arc current and the frequency of the current interruption can be calculated [5].

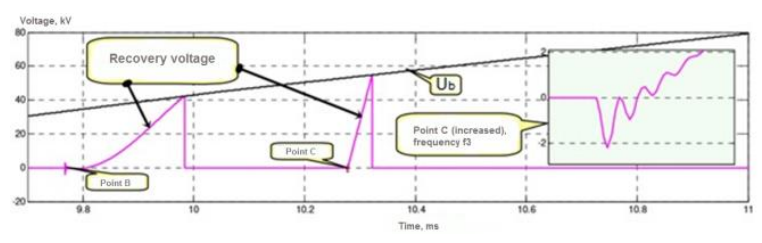

Fig. 2. Voltage and breakdown characteristic of VCB, where $\mathrm{U}_{\mathrm{b}}$ is the breakdown characteristic.

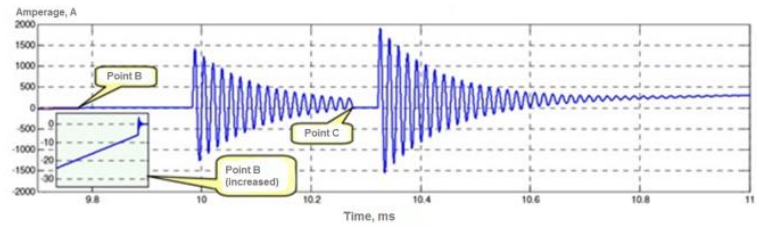

Fig. 3. Current cross-section and re-ignition frequency.

At point "B" of the lower diagram, the current is interrupted until the real zero value of the current $6 \mathrm{~A}$ is reached, which is not visible on the current waveform from Fig. 3. While point "B" is the cut-off current of the industrial frequency, point " $\mathrm{C}$ " is the cut-off current of the high frequency. After this interruption of the current, the gap voltage will be restored again, coinciding with the frequency "fl". As for interrupting the highfrequency current, the oscillating frequency "f3" is superimposed on the recovering voltage, which in our case is about $1700 \mathrm{kHz}$ or $1.7 \mathrm{MHz}$. The recovering voltage, which is superimposed on the frequency "f3", is increased in the upper right corner of Figure 2.

At the time of the current cutoff, accompanied by repeated re-activation (point "B" in Fig. 4), highfrequency currents can be induced on the other two phases. Since all three poles opened at the same time, the other two phases still have current flowing through the arc. Then, in some special cases with a strong capacitive/inductive coupling between the phase circuits, the above-mentioned induced high-frequency currents can occur when other phases break in the adjacent conductor, in which a significant current is still flowing, which is some millisecond away from their natural zero [6]. If the induced high-frequency currents reach an amplitude greater than the amplitude of the industrialfrequency current, then current zeros are formed, and this current zero is "artificial", but, nevertheless, real, and not virtual. The device can take advantage of the disadvantage of this effect by breaking the current a few milliseconds before its natural zero. In such cases, the interrupt current can be tens or even hundreds of amperes, and the associated overvoltages, as already explained, are very high. Of course, there is nothing 
surprising in extremely high transient overvoltages due to the very rapid occurrence of breakdown in the vacuum interrupter.

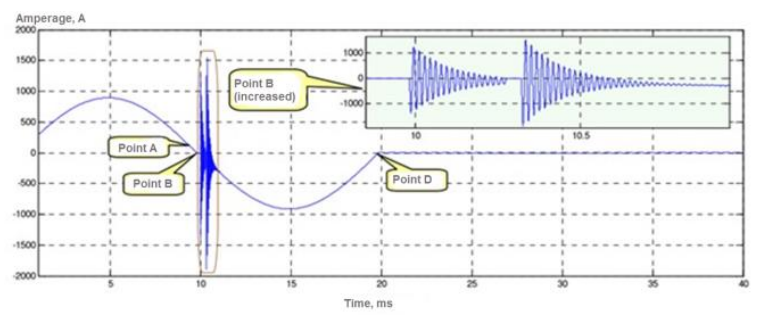

Fig. 4. The value of the VCB current when triggered.

Evidently, switching in electrical grids leads to transients, which, in turn, can be accompanied by significant overvoltages and current surges, which leads to a decrease in the resource of the contact system.

\subsection{Modeling of VCB electrodes}

It is known that the VCB contact electrodes made of arc resistance contact pads connected to the protrusion of the disk base, the protrusion base is made in the form of bulk segment height from 1 to $10 \mathrm{~mm}$, and the base and the pad covers the clip that holds the disc protrusion and arc resistance contact strip, ensuring the rigidity of the switch contact, and the ratio of the length of the segment to the base disk is in the range of 0.3-0.45 $\mathrm{mm}$.

In our research [7], the goal was to reduce the surface temperature of the mobile and stationary contacts in the processes of closing and opening. For this purpose, arcresistant pads made of heat-resistant hardened alloys based on $\mathrm{Mo}, \mathrm{W}$, and $\mathrm{Nb}$ were used as electrodes. However, due to the complexity of the manufacturing technology and a number of electrical parameters, the best properties were shown by single-crystal electrodes, in particular those made of copper $(\mathrm{Cu})$ and nickel $(\mathrm{Ni})$, which have a cubic structure. After that, it was made as a lining for stationary electrode - an electrode made of copper with cubic structure (grown from the melt by the Bridgman method) and specific direction [111], (Fig. 5).

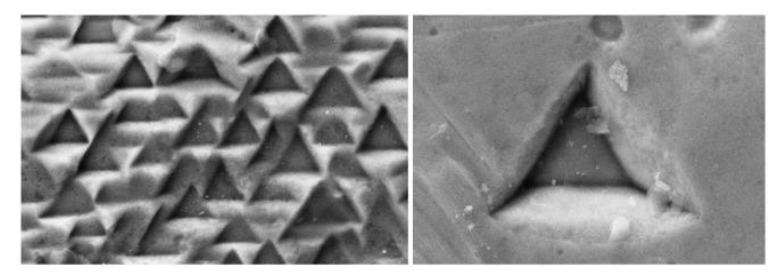

Fig. 5. The structure of the single crystal Ni (111).

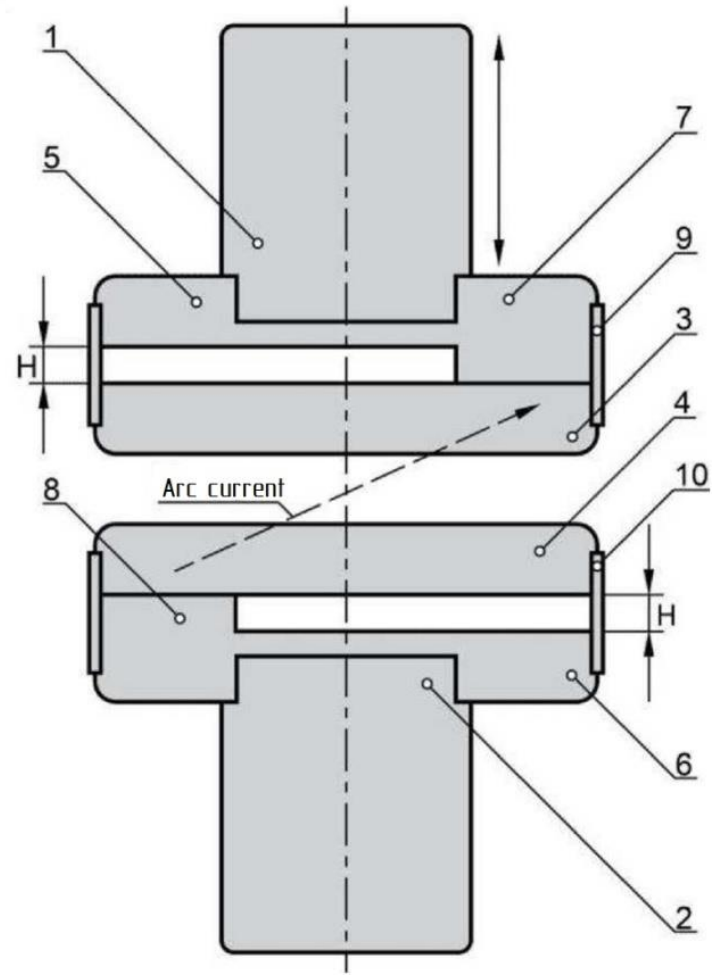

Fig. 6. Terminal device, where the 1 - movable switching contact; 2 - stationary switching contact; 3,4 - arc resistance contact strip of hardened high-temperature single-crystal alloys; 5, 6 - disc basis of contact; 7, 8 - lug disc base; 9, 10 clip.

From Fig. 6, it can be seen that when the electrodes are opened, the arc current acquires an inclined trajectory with respect to the plane of contact of the contact pads 3 and 4 .

Using the COMSOL Multiphysics software package, the processes occurring in a vacuum chamber using overlays made of single-crystal copper electrodes in the opening mode at a voltage of $35 \mathrm{kV}$ were simulated (Fig. 7). Plasma in the inter-contact space 11, despite the sharply inhomogeneous electric field created by the use of a rod 12 acting as a geometric defect on one of the overlays and an arc-resistant contact plate 3 made of heat-resistant hardened single-crystal alloys.

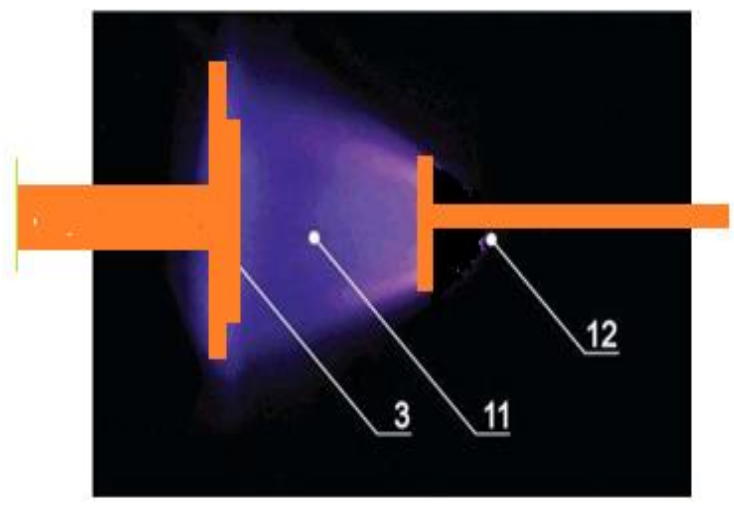

Fig. 7. Electric arc distribution in the contact gap. 


\section{Conclusions}

- The possibility of increasing the energy characteristics of vacuum circuit breakers by using single-crystal electrodes of copper, nickel, and iron as overlays is shown.

- Experimental studies show that the change in electric field distribution, namely (exception streamer corona plasma), the creation of an inhomogeneous field, increases reliability and switching online contact system VCB.

- The possibility of using explosives in power grids with a voltage of more than $110 \mathrm{kV}$ is shown.

\section{References}

1. L. Liu, Zh. Yuan, Sh. Liu, Experiment research of post-arc current and cathode spots distribution in medium-high frequency vacuum arc, Physics of Plasmas (2020)

2. G. Daigneault, et all, Comparing direct and synthetic tests for interruption of line-charging capacitive current, Power Delivery, IEEE Transactions on, 16, 409-414 (2001)

3. V.D. Sluis, Transients in Power Systems (Arnhem: John Wiley \& Sons Ltd, 2001)

4. A. Kravchenko, V. Metelsky, Vacuum load switches of foreign manufacturers, Electrician, 3, 14-17 (2013)

5. R.P.P. Smeets, Low-current behavior and current chopping of vacuum arcs, $\mathrm{PhD}$, Technical University of Eindhoven, Eindhoven, The Netherlands (1987)

6. P. Picot, Vacuum switching (France: Schneider, 2000)

7. Contact device for a vacuum circuit breaker with arc-resistant plates made of anisotropic materials, Patent RU 197821 U1

8. D.E. Shevtsov, D.A. Pavlyuchenko, V.A. Lavrinovich, V.G. Shalnev, Development of a simulation model of a 6 (10) $\mathrm{kV}$ synchronous vacuum circuit breaker taking into account the real characteristics of the device, Elektro., Electrical engineering, electric power industry, electrical industry, 1, 38-44 (2017)

9. H.W. Dommel, Digital Computer Solution of Electromagnetic Transients in Single and Multiple Grids, IEEE Transactions on Power Apparatus and Systems, PAS\#88 (4), 388-399 (1969)

10. V.V. Bazutkin, K.P. Kadoma, M.V. Kostenko, Y.A. Mikhailov, Overvoltage in electrical systems and protection against them: textbook, For higher education institutions (St. Petersburg: Energoatomizdat, St. Petersburg. otd-ie, 320, 1995)

11. I.A. Lebedev, E.V. Prokhorenko, Investigation of the possibility of creating a vacuum circuit breaker for synchronous shutdown of unloaded transformers, Elektro., 3, 40-44 (2011)
12. K.P. Kadomskaya, E.S. Nesgovorov, L.V. Petrakova, V.S. Ponomarev, Limiting internal overvoltages by controlling the switching moments of switches, Electricity, 9, 10-13 (1969)

13. S.M. Apollonsky, A.E. Kozyaruk, Yu.V. Kuklev, Tests and control systems of electrical devices: a textbook for universities (St. Petersburg: Troitsky Bridge, 326, 2015)

14. GOST 27.002-2015, Reliability in technology, Basic concepts, Terms and definitions, Moscow: Izd-vo standartov, 24 (2015)

15. V.P. Kalyavin, L.M. Rybakov, Reliability and diagnostics of elements of electrical installations: textbook, handbook for universities (St. Petersburg: Elmore, 336, 2009)

16. A.A. Chunikhin, Electric apparatuses, General course: textbook for universities (3rd ed., reprint. and add., M.: Energoatomizdat, 720, 1988)

17. A.V. Vinogradov, et all, Analysis of the time of interruptions in the power supply of rural consumers and methods of reducing it by monitoring the technical condition of power transmission lines, Vestnik RESKH, 2 (27), 3-11 (2017) 